\title{
Effects of surgical clipping and endovascular embolization on the recovery of oculomotor nerve paralysis caused by posterior communicating artery aneurysm
}

\author{
*Kang $\mathrm{Hu} B S c$, *Genping Cai $B S c$, Liang Fu $B S c$, Lili Huang $B S c$, Wei Huang $P h D$, Wenhao \\ Wang $B S c$, Changchun Liu $B S c$ \\ *These authors contributed equally to this work and are Co-First Author
}

Department of Neurosurgery, The Affiliated Dongnan Hospital of Xiamen University, Fujian, China

\begin{abstract}
Background and Objectives: Our study aimed to explore the therapeutic effects of surgical clipping and endovascular embolization on the recovery of oculomotor nerve paralysis (ONP) caused by posterior communicating artery aneurysm (PcomAA). Methods: The clinical data of patients with intracranial PcomAA and ONP were retrospectively analyzed. All patients were treated with surgical clipping or endovascular embolization, then followed up for no less than 12 months. Logistic regression analysis was performed to analyze the potential risk factors influencing ONP recovery. Results: Among 128 patients of ONP caused by PcomAA, 96 patients were treated with surgical clipping and 32 patients with endovascular embolization, respectively. Time from initial ONP onset to complete or partial recovery was $85.3 \pm 36.8$ days for patients receiving surgical clipping, and $135.7 \pm 41.3$ days for patients treated with endovascular embolization. The recovery rate was 94 (97.9\%) in the surgical clipping group and $22(68.8 \%)$ in the endovascular embolization group, and significant difference was shown between the two groups $(\mathrm{P}<0.001)$. Logistic regression analyses demonstrated that the complete or partial recovery of ONP in the surgical clipping group was significantly better than that in the endovascular embolization group (OR, 5.582; 95\% CI, 2.023-15.405; $\mathrm{P}<0.001)$. Moreover, time from initial symptom onset to receiving treatment also affect ONP recovery (OR, $0.893 ; 95 \%$ CI, 0.820-0.972; $\mathrm{P}=0.009)$.

Conclusion: Surgical clipping was superior to endovascular embolization in the recovery of ONP caused by PcomAA, and patients who received early intervention could result in better ONP recovery.
\end{abstract}

Keywords: Posterior communicating artery aneurysm; oculomotor nerve palsy; surgical clipping; endovascular embolization

\section{INTRODUCTION}

Posterior communicating artery aneurysms (PcomAA) usually indicate an aneurysm at the bifurcation of the internal carotid artery-posterior communicating artery. It is the second common type of intracranial aneurysm, accounting for $29.3 \%$ of ruptured aneurysms and $24.4 \%$ of unruptured aneurysms. ${ }^{1}$

Unilateral oculomotor nerve palsy (ONP) is a common symptom caused by compression of PcomAA. There is one case of ONP in every five PcomAA patients, and almost 50\% of ONP patients have PcomAA with subarachnoid hemorrhage (SAH). Unilateral ONP has been found to be the only neurological sign in some patients with PcomAA. Direct compression or pulsatile effect of the aneurysm could result in partial or complete ONP. Majority of the studies suggest that pulsatile stimulation of PcomAA could be the main pathogenic effect of ONP. Other causes are direct compression of aneurysms to the oculomotor nerves, and the effect of the aneurysmal SAH. Prevention of aneurysm rupture could help the recovery of ONP symptoms (complete or incomplete), such as ipsilateral ptosis, ophthalmoplegia, diplopia, and mydriasis. ${ }^{2}$

Address correspondence to: Changchun Liu and Wenhao Wang, Department of Neurosurgery, The Affiliated Dongnan Hospital of Xiamen University, Zhangzhou, Fujian, China. E-mail: liuchangchun_med@outlook.com (CC Liu), 741324739@qq.com (WH Wang)

Date of Submission: 13 February 2021; Date of Acceptance: 26 April 2021

https://doi.org/10.54029/2021vnw 
Treatment strategies for PcomAAin combination with ONP include microsurgical clipping and intravascular coil therapy. Microsurgical clipping of the PcomAA relieve pulsation and compression, which promote the healing of the ONP. ${ }^{3}$ Aneurysm intravascular coil embolization also promotes the recovery of oculomotor function, the underlying mechanism may be related to the elimination of aneurysm pulsation. ${ }^{4}$ Patients who received surgical clipping or endovascular embolization could achieve complete or partial recovery of the ONP. ${ }^{5,6}$ However, the effects of the two therapeutic methods on the ONP recovery were still controversial. In this study, we aimed to investigate the therapeutic effects of surgical clipping and endovascular embolization on the recovery of ONP caused by PcomAA.

\section{METHODS}

\section{Patients}

This single-center retrospective cohort study analyzed clinical data of patients with ONP caused by PcomAA, who were treated in the Department of Neurosurgery, the Affiliated Dongnan Hospital of Xiamen University between June 2015 and August 2019. Inclusion criteria were: 1) Patients were diagnosed as ONP caused by PcomAA and all aneurysms were confirmed by digital subtraction angiography (DSA) and/or computed tomography angiography (CTA). 2) Patients were treated with craniotomy surgical clipping or endovascular embolization. Exclusion criteria were: 1) Diabetic ophthalmoplegia; 2) Tolosa-Hunt Syndrome; 3) Postoperative follow-up less than 12 months. This study was approved by the Ethics Committee of the Affiliated Dongnan Hospital of Xiamen University, and the informed consent was waived by the Ethics Committee.

\section{Treatments}

For both unruptured and ruptured aneurysms, the treatments were recommended per the criteria of International Study of Unruptured Intracranial Aneurysms. ${ }^{7}$ The potential risk and difficulties of surgical clipping and embolization coiling were assessed by the neurosurgeons and neuro-interventional physicians based on the characteristics of each patient. All patients and/or their family members then selected the treatment strategy after explanation of the surgical indications and procedures. The patients were divided into the surgical clipping group and the endovascular embolization group. For patients with $\mathrm{SAH}$ at diagnosis, the treatment regimen included intravenous drip of $50 \mathrm{mg}$ nimodipine every 12 hours (ivgtt $\mathrm{q} 12 \mathrm{~h}$ ) and tranexamic acid 2.0 (ivgtt q12h) throughout the time from admission to surgery.

Surgical clipping: The unilateral (affected side) standard pterional approach was conducted for surgical clipping. All operations were performed by the same surgeon who specialized in surgical clipping of PcomAA for more than 5 years. The patient was in a supine position with the head facing the opposite side. When the standard pterional approach was used for craniotomy aneurysm clipping on the affected side, the sphenoid ridge was removed to obtain the operative space. The lateral fissure was separated, the optic chiasm cistern and the internal carotid artery cistern were opened, the cerebrospinal fluid was gradually released, and the proximal end of the internal carotid artery was exposed for temporary occlusion. The aneurysm neck was fully dissociated, the appropriate aneurysm clip (B. Braun Melsungen Ag., Germany) was selected according to the width of the aneurysm neck, and the surrounding posterior communicating artery and anterior choroidal artery were protected. For patients with hydrocephalus, lamina terminalis fenestration was performed during the operation. Because the apex of aneurysm capsule was generally tightly attached to the oculomotor nerve, we only clamped the aneurysm neck and did not deliberately separate the adhesion to prevent the damage of oculomotor nerve.

Endovascular embolization: A 6F guide catheter (Chaperon, Boston Company, USA) was usually placed in the affected internal carotid artery. Under the path diagram, the microcatheters (Echelon-10, EV3 Company, USA) were carefully inserted into the aneurysm lumen. After the aneurysm was confirmed by angiography, the spring coils with appropriate size (Axium, EV3 Company, USA) were selected. The multiple spring coils filled the aneurysm cavity in turn, until the aneurysm was no longer visible with angiography. For wideneck aneurysms, assistance of stent (solitaire, EV3 Company, USA or enterprise, Codman Company, USA) was also required. If spring coil with stent assistance was used for embolization, oral administration of $100 \mathrm{mg}$ aspirin and 75 $\mathrm{mg}$ clopidogrel were given daily from three days before operation until three months after operation. All these procedures were conducted by the same neuro-interventional physician who specialized in intravascular embolization and stenting for more than 5 years, with the assistance of an anesthesiologist. 


\section{Data collection}

The potential variables that might affect the recovery of ONP were collected, including age, gender, size of aneurysm, hypertension, hyperlipidemia, presence or absence of preoperative $\mathrm{SAH}$, the degree of preoperative ONP, time from first onset of ONP symptoms to treatment, different treatment methods and postoperative recovery time. The maximum diameter of aneurysm was also noted. Hypertension was defined as systolic blood pressure (SBP) $\geq 140 \mathrm{mmHg}$ and/or diastolic blood pressure (DBP) $\geq 90 \mathrm{mmHg}$. Hyperlipidemia was defined as the level of blood total cholesterol, triglyceride or low-density lipoprotein cholesterol was higher than upper limit of normal value. The patient received head CT/MRI and lumbar puncture to determine the presence or absence of preoperative $\mathrm{SAH}$ as required. The clinical status of aneurysmal SAH was graded based on the Hunt-Hess classification.

\section{Follow-up}

All patients were followed up at 1, 3, 6 and 12 months after surgery. Postoperative neurological assessment was performed by the same senior neurologist who were blinded to the treatment assignment. The curative effects of both surgical procedures were followed up by imaging. For patients who underwent craniotomy, CTA was performed 7-10 days after surgery to evaluate the occlusion of the aneurysm. For patients who underwent endovascular embolization, DSA was performed immediately after surgery and 6-12 months after surgery to evaluate the effect of interventional therapy.

The definition of ONP and the recovery evaluation were as below: The clinical manifestations of ONP included blepharoptosis, ophthalmoplegia, diplopia, dilated pupils of the affected side, and absence of direct and indirect light reflex. ${ }^{8}$ If one or more of the listed symptoms was seen, the patient was deemed to have partial ONP recovery. When all the abovementioned symptoms were not seen, the ONP was deemed to have recovered completely. If one or more symptoms still existed, it was regarded as partial recovery. ONP recovery was divided into complete recovery, partial recovery, no recovery and symptoms aggravation according to patient's postoperative condition. Postoperative recovery time was defined as the period since surgical clipping or endovascular embolization to ONP recovery.

\section{Statistical analysis}

All statistical analyses were performed using the SPSS19.0 (IBM, Armonk, NY, USA) software. The distribution of continuous data was performed using the Kolmogorov-Smirnov test. Normally distributed continuous data were shown as mean \pm standard deviation (SD) and compared using the Student $t$ test. Non-normally distributed data were presented as median (range) and analyzed with the Mann-Whitney U test. Categorical data were presented as proportion and analyzed with the chisquare test or Fisher's exact test, if appropriate. Logistic regression analyses were performed to explore the association between clinical variables and ONP recovery (Entered method). P values $<0.05$ were considered statistically significant.

\section{RESULTS}

Baseline data of patients between the surgical clipping group and the endovascular embolization group.

Figure 1 presented the study flowchart, between June 2015 and August 2019, 1,227 patients with intracranial aneurysms were treated in the Department of Neurosurgery, The Affiliated Dongnan Hospital of Xiamen University. Of them, 405 cases were confirmed to have PcomAA. Two patients in the surgical clipping group were lost to follow up because they lived far from the hospital and achieved complete recovery about 6 months after surgery. Another patient in the endovascular embolization group died from traffic accident that occurred 4 months after treatment. Finally, 128 patients with PcomAA complicated by ONP met the inclusion criteria for final analysis, in which 32 patients underwent endovascular embolization and 96 patients underwent surgical clipping. All patients were followed-up for at least 12 months. There were no significant differences in terms of age, gender, comorbid hypertension or hyperlipidemia between the two treatment groups (all $\mathrm{P}>0.05$ ). The mean diameter of PcomAA was $7.82 \pm 1.70$ and $7.71 \pm 1.63 \mathrm{~mm}$ in the surgical clipping group and endovascular embolization group, respectively. Forty-seven (49.0\%) patients and $15(46.9 \%)$ patients had complete ONP before treatment in the surgical clipping group and the endovascular embolization group, respectively. Among the 96 patients in the surgical clipping group, $49(51.0 \%)$ patients had SAH, while, 16 $(50.0 \%)$ patients of the embolization group had $\mathrm{SAH}$ at diagnosis. Time from occurrence of initial ONP symptoms to receiving treatment was 11.21 


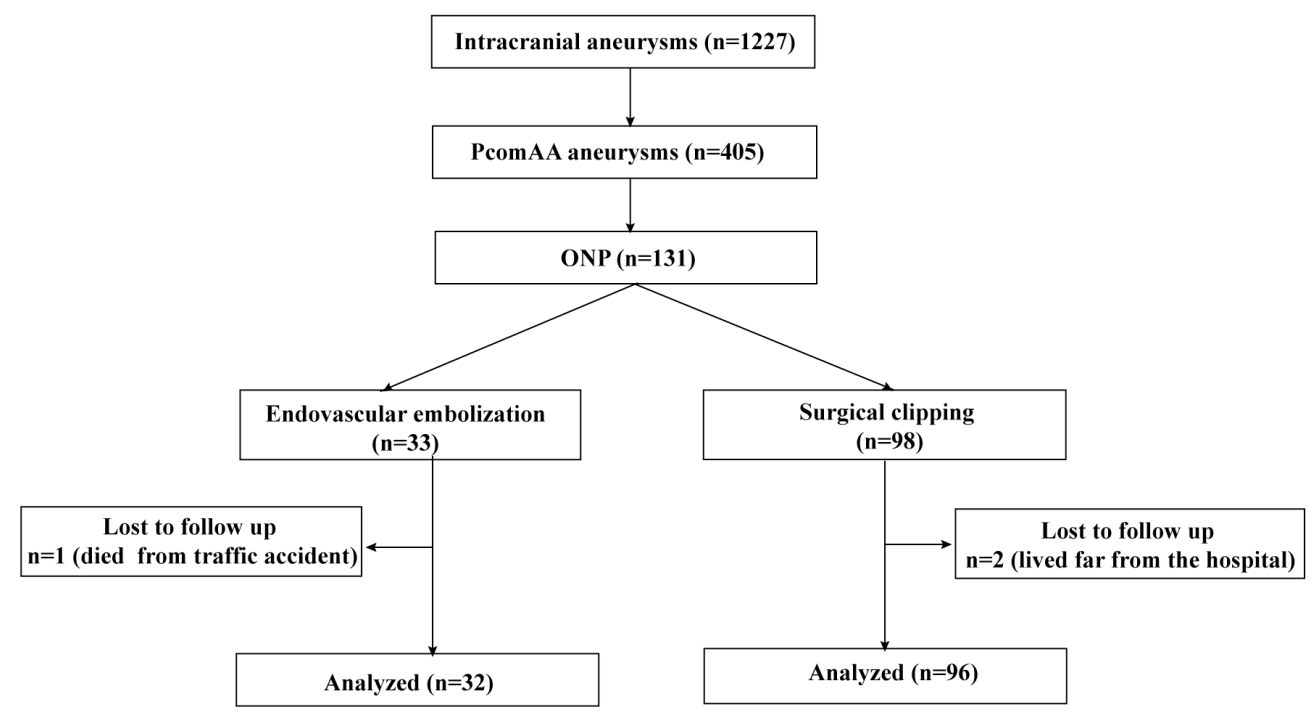

Figure 1. Study flowchart.

\pm 8.76 and $10.53 \pm 9.17$ days in the surgical clipping group and the endovascular embolization group, respectively. In addition, patients with SAH were usually admitted to hospital due to severe and sudden headaches, therefore, the time from occurrence of ONP symptoms was significantly shorter for those with SAH $(n=65)$ than those $(\mathrm{n}=63)$ without SAH $(8.12 \pm 5.68$ days versus $13.62 \pm 8.35$ days; $\mathrm{P}<0.001)$. As shown in Table 1, the baseline data of the two groups of patients was comparable (all $\mathrm{P}>0.05$ ).

\section{Postoperative follow-up}

Time to complete or partial recovery after treatment was $85.3 \pm 36.8$ days and $135.7 \pm 41.3$ days for patients who underwent surgical clipping and patients receiving endovascular embolization, respectively. In addition, the recovery rate was $97.9 \%$ in the surgical clipping group and $68.8 \%$ in the endovascular embolization group, and the differences between the two groups was statistically significant $(\mathrm{P}<0.001)$. During the 12-month postoperative follow-up period, 108

Table 1: Comparison of clinical data between the surgical clipping group and embolization group

\begin{tabular}{lccc}
\hline Variables & $\begin{array}{c}\text { Embolization group } \\
(\mathbf{n = 3 2})\end{array}$ & $\begin{array}{c}\text { Clipping group } \\
(\mathbf{n = 9 6})\end{array}$ & P value \\
\hline Age (year) & $52.8 \pm 10.2$ & $53.6 \pm 11.7$ & 0.730 \\
Gender, male, n (\%) & $17(53.1 \%)$ & $43(44.8 \%)$ & 0.413 \\
Diameter of aneurysm (mm) & $7.71 \pm 1.63$ & $7.82 \pm 1.70$ & 0.744 \\
Hypertension, n (\%) & $18(56.3 \%)$ & $45(46.9 \%)$ & 0.358 \\
Hyperlipidemia, n (\%) & $16(50.0 \%)$ & $44(45.8 \%)$ & 0.683 \\
Time from occurrence of ONP symptoms & $10.53 \pm 9.17$ & $11.21 \pm 8.76$ & 0.714 \\
to receiving treatment (days) & $16(50.0 \%)$ & $49(51.0 \%)$ & 0.919 \\
SAH, n (\%) & & & 0.838 \\
Preoperative ONP symptoms & $15(46.9 \%)$ & $47(49.0 \%)$ & \\
Complete ONP, n (\%) & $17(53.1 \%)$ & $49(51.0 \%$ & \\
Incomplete ONP, n (\%) & &
\end{tabular}

Data are shown as mean $\pm \mathrm{SD}$ or $\mathrm{n}(\%)$.

Note: $\mathrm{ONP}=$ oculomotor nerve palsy, $\mathrm{SAH}=$ subarachnoid hemorrhage, $\mathrm{SD}=$ standard deviation. 
patients were recovered from the preoperative ONP symptoms, of which 99 patients had complete recovery (89 patients in the surgical clipping group and 10 patients in the endovascular embolization group); 17 patients had partial recovery (5 patients in the surgical clipping group and 12 patients in the endovascular embolization group). The ONP in 12 patients ( 2 patients in the surgical clip group and 10 patients in the endovascular embolization group) did not recover. For the postoperative recovery of ONP symptoms throughout the 12-month follow-up period, blepharoptosis has best recovery, recovery of ophthalmoplegia and diplopia were relatively less, and recovery of mydriasis and light reflex were even less. The postoperative recovery of ONP was shown in Table 2.

\section{Logistic regression analyses}

The potential risk factors, including age, sex, aneurysm diameter, preoperative complete ONP, time from occurrence of initial ONP symptom to receiving treatment and treatment strategy were entered to multivariate logistic regression analyses. ONP recovery in the surgical clipping group was significantly better than that in the endovascular embolization group (OR, 5.582; 95\% CI, 2.023-15.405; $\mathrm{P}<0.001)$. Time from occurrence of initial ONP symptom to receiving treatment also showed a significant effect on the ONP prognosis (OR, 0.893; 95\% CI, 0.820-0.972; $\mathrm{P}=0.009$ ), suggesting that patients who received early treatment of PcomAA could achieve better ONP recovery. The results of multivariate analyses were shown in Table 3.

\section{Postoperative complications}

There were no treatment-related deaths in either group. There were 5 patients experienced cerebral vasospasm, including 1 patient in the endovascular embolization group and 4 patients in the surgical clipping group. One patient had

Table 2: Comparison of postoperative data between the surgical clipping group and endovascular embolization group

\begin{tabular}{|c|c|c|c|}
\hline Variables & $\begin{array}{c}\text { Embolization group } \\
(\mathrm{n}=32)\end{array}$ & Clipping group (n=96) & $P$ value \\
\hline $\begin{array}{l}\text { Recovery time, days, } \\
(\text { mean } \pm \text { SD })\end{array}$ & $135.7 \pm 41.3$ & $85.3 \pm 36.8$ & $<0.001$ \\
\hline Recovery rate $\mathrm{n}(\%)$ & $22(68.8 \%)$ & $94(97.9 \%)$ & $<0.001$ \\
\hline Complete recovery & 10 & 89 & \\
\hline Partial recovery & 12 & 5 & \\
\hline No recovery & 10 & 2 & \\
\hline \multicolumn{4}{|l|}{ Postoperative symptoms } \\
\hline Blepharoptosis & $\begin{array}{c}7 \text { partial recovery; } \\
5 \text { recovery; } \\
0 \text { no recovery }\end{array}$ & $\begin{array}{c}1 \text { partial recovery; } \\
4 \text { recovery; } \\
0 \text { no recovery }\end{array}$ & \\
\hline Ophthalmoplegia & $\begin{array}{c}4 \text { partial recovery; } \\
1 \text { no recovery; } \\
7 \text { recovery }\end{array}$ & $\begin{array}{c}1 \text { partial recovery; } \\
1 \text { no recovery; } \\
3 \text { recovery }\end{array}$ & \\
\hline Diplopia & $\begin{array}{l}5 \text { partial recovery; } \\
2 \text { no recovery; } \\
5 \text { recovery }\end{array}$ & $\begin{array}{c}1 \text { partial recovery; } \\
2 \text { no recovery; } \\
2 \text { recovery }\end{array}$ & \\
\hline Mydriasis & $\begin{array}{c}5 \text { partial recovery; } \\
3 \text { no recovery; } \\
4 \text { recovery }\end{array}$ & $\begin{array}{c}3 \text { partial recovery; } \\
1 \text { no recovery; } \\
1 \text { recovery }\end{array}$ & \\
\hline Disappeared light reflex & $\begin{array}{c}4 \text { partial recovery; } \\
4 \text { no recovery; } \\
4 \text { recovery }\end{array}$ & $\begin{array}{c}1 \text { partial recovery; } \\
3 \text { no recovery; } \\
1 \text { recovery }\end{array}$ & \\
\hline
\end{tabular}

Note: $\mathrm{SD}=$ standard deviation.

No recovery referred to the symptoms compared with those before treatment. 
Table 3: Logistic regression model was preformed to evaluate the potential risk factors of postoperative ONP recovery induced by $\mathbf{P c o m A A}$

\begin{tabular}{lccc}
\hline Risk factors & OR & 95\% CI & P value \\
\hline Age & 1.217 & $0.873-1.696$ & 0.246 \\
Sex & 0.760 & $0.439-1.317$ & 0.328 \\
Diameter of aneurysm & 0.816 & $0.396-1.680$ & 0.581 \\
$\begin{array}{l}\text { Preoperative complete ONP } \\
\text { Time from occurrence of initial ONP symptom to } \\
\text { receiving treatment (days) }\end{array}$ & 0.812 & $0.619-1.090$ & 0.172 \\
$\begin{array}{l}\text { Treatment strategy (surgical clipping versus } \\
\text { embolization therapy) }\end{array}$ & 0.893 & $0.820-0.972$ & 0.009 \\
\hline
\end{tabular}

Note: $\mathrm{ONP}=$ oculomotor nerve palsy; $\mathrm{OR}=$ odds ratio; $\mathrm{CI}=$ confidence interval.

the partial hemiplegia in the surgical clipping group, one patient had aphasia in the endovascular embolization group. A total of 18 patients had hydrocephalus after treatment (6 patients in the endovascular embolization group and 12 patients in the surgical clipping group). One patient in each group required ventriculo-peritoneal shunt after multiple lumbar punctures to release the blood cerebrospinal fluid. All the complications partially or completely recovered. The postoperative complications of the two groups were listed in Table 4.

\section{DISCUSSION}

Our study explored which therapeutic method was more beneficial for patients with ONP induced by PcomAA. The results demonstrated that postoperative ONP recovery rate in patients with surgical clipping was significantly superior to those receiving endovascular embolization. In addition, a shorter duration from the onset of ONP to receiving treatment was independently associated with better ONP recovery.

Endovascular embolization and surgical clipping had their own pros and cons for the treatment of patients with PcomAA with ONP. ${ }^{9}$ Previous study proposed that compared with traditional craniotomy, endovascular embolization could also restore the oculomotor nerve function of patients in PcomAA with ONP. ${ }^{4}$ The oculomotor nerve is prone to insults resulting in ONP-related symptoms. Even in patients with intracranial aneurysms only, when the oculomotor nerve was pulled to fully expose the aneurysm, various degrees of ONP may occur after surgery. Previous studies had shown that the effect of endovascular embolization was not inferior to microsurgery, which was also conducive to the recovery of oculomotor function., ${ }^{2,9}$

The endovascular embolization by forming a thrombus in the aneurysm, could alleviate the insult from the aneurysm pulsation, reducing the pulsating effect of the aneurysm on the nerve, could partially or completely lead to recovery of the oculomotor nerve function..$^{10,11}$ On the other hand, although the craniotomy results in injury to the patient, the clipping of PcomAA could reduce the compression of the aneurysm to the oculomotor nerve, decrease and block the aneurysm pulsations, prevent and control the aneurysm rebleeding, thereby improving the ONP symptoms and quality of life. ${ }^{12,13}$ Previous studies have shown that the endovascular embolization was not ideal for the relief of insult from aneurysm. The coil implanted may occasionally aggravate the pulsation effect. ${ }^{5,12}$ The reasons for better ONP recovery in the clipping

Table 4: Postoperative complications

\begin{tabular}{lcc}
\hline Complication & Embolization group $(\mathbf{n}=32)$ & Clipping group (n=96) \\
\hline Significant cerebral vasospasm & 1 & 4 \\
Partial hemiplegia & 0 & 1 \\
Aphasia & 1 & 0 \\
Hydrocephalus & 6 & 12 \\
Ventriculo-peritoneal shunt & 1 & 1 \\
\hline
\end{tabular}


group than in the embolization group may be as follows: 1) craniotomy could remove the local hematoma, while endovascular embolization would just allow the hematoma to be absorbed naturally; 2) aneurysm body may still enlarge after endovascular embolization, while craniotomy would shrink the aneurysm body immediately; 3) a small amount of blood extravasation may occur after endovascular embolization..$^{6,14,15}$

Previous study suggested that, compared to surgical clipping, patients with older age and other comorbidities seemed to benefit more from the endovascular embolization treatment. ${ }^{1}$ Our results showed no significant differences in terms of age, aneurysm size, degree of ONP symptom at baseline, time interval between initial ONP symptom onset and treatment, and preoperative SAH between the two groups. Multivariate analyses results indicated that time interval between initial ONP symptom onset and treatment modality were independently associated with postoperative ONP recovery. Compared with the endovascular embolization group, the interval between initial ONP symptom onset and treatment was longer in the surgical clipping group, but this did not affect the beneficial effect of surgical clipping.

Previous studies have reported a complete recovery rate of ONP that ranged from $32 \%$ to $85 \%$. Under early surgical treatment, the complete recovery rate could achieve $88 \% .{ }^{14}$ On the other hand, complete recovery rate with embolization treatment ranged between $0 \%$ and $50 \% .^{16,17}$ Regardless of the treatment options, patients with partial ONP at the time of admission had the higher complete recovery rate compared with those with complete ONP at baseline. ${ }^{2,18}$ The complete recovery rate of patients receiving surgical clipping was much higher than that reported in patients with endovascular therapy, suggesting that recovery mechanism of embolization treatment may be associated with thrombosis within the aneurysm capsule after embolization, which result in reduced pulsation effect to the oculomotor nerve. However, the pulsatile effect of the aneurysm was still present. Surgical clipping could simultaneously eliminate the effects derived from two mentioned mechanisms (compression and pulsation), both of which could be damaging to the oculomotor nerve. ${ }^{2,15} \mathrm{We}$ believed that the causes of ONP induced by PcomAA were multifactorial, including the combined effects from the mass effect of aneurysm compression and the pulsation conducted through aneurysm pulsation. Pulling the oculomotor nerve to expose the aneurysm neck and/or oculomotor nerve could also result in various degrees of ONP after surgery. The clipping operations might damage the oculomotor nerve, and further aggravate the ONP symptoms. Therefore, only the aneurysm neck was clamped during the operation, and the aneurysm sac was not be dissociated from the oculomotor nerve, which was consistent with the viewpoints of previous studies..$^{14,19}$

Early treatment effects for PcomAA in combination with ONP and the degree of postoperative ONP recovery had been assessed in several studies, however, the results were somewhat controversial. Yang et al. reported that ONP recovery was better when the aneurysm was treated earlier. ${ }^{20} \mathrm{We}$ observed that the time interval between initial ONP symptom onset and treatment was independently associated with postoperative ONP recovery, which was also consistent with the results from $\operatorname{Tan}^{19}$ and Chang ${ }^{21}$, but differed from the findings from Hanse et al. ${ }^{22}$ Post-operative treatment options also differed in patients with ONP caused by PcomAA. Our patients with no ONP improvement had a follow-up period no less than 1 year. Previous studies found that improvements of ONP symptoms always occurred within the first year after intervention., ${ }^{2,23}$ In our study, patients with no improvement of ONP symptoms continued to receive nutritional neurotherapy, traditional Chinese medicine or acupuncture treatment, but these symptoms persisted.

Our study also had some limitations. First, this was a single-center retrospective cohort, without the benefit of randomized allocation of treatment. The sample size was relatively small and postoperative follow-up period was only 1 year. Multicenter, randomized controlled trials with large sample sizes is required for direct comparisons between surgical clipping and intravascular embolization for the treatment of ONP induced by PcomAA. Second, direct nerve damage caused by hemorrhagic shock from aneurysm rupture was one of the possible causes of ONP, and patients with this kind of trauma were less likely to achieve complete recovery. Nevertheless, it was impossible to differentiate ONP-mass oppression, aneurysm pulsation, and shock effect induced by aneurysm rupture, respectively. Nevertheless, the hemorrhagic shock effect caused by aneurysm rupture could not be considered as the principal cause of ONP, due to the presence of many patients with non-ruptured aneurysms.

In conclusion, our study demonstrated that 
the ONP symptoms caused by PcomAA could be alleviated through either surgical clipping or endovascular treatment. However, the recovery rate and degree of ONP recovery in patients receiving clipping surgery was better compared to those with endovascular treatment, irrespective of patient's the aneurysm size and presence of SAH.

\section{DISCLOSURE}

Financial support: This research received grant from General Program of National Natural Science Foundation of China (No. 2018J01152).

\section{Conflict of interest: None.}

\section{REFERENCES}

1. Golshani K, Ferrell A, Zomorodi A, Smith TP, Britz GW. A review of the management of posterior communicating artery aneurysms in the modern era. Surg Neurol Int 2010;1:88.

2. Patel K, Guilfoyle MR, Bulters DO, et al. Recovery of oculomotor nerve palsy secondary to posterior communicating artery aneurysms. $\mathrm{Br} J$ Neurosurg 2014;28(4):483-7.

3. Güresir E, Schuss P, Seifert V, Vatter H. Oculomotor nerve palsy by posterior communicating artery aneurysms: influence of surgical strategy on recovery. J Neurosurg 2012;117(5):904-10.

4. Sheehan MJ, Dunne R, Thornton J, Brennan P, Looby S, O'Hare A. Endovascular repair of posterior communicating artery aneurysms, associated with oculomotor nerve palsy: A review of nerve recovery. Interv Neuroradiol 2015;21(3):312-6.

5. Gao G, Gu DQ, Zhang Y, et al. Comparison of the efficacy of surgical clipping and embolization for oculomotor nerve palsy due to a posterior communicating artery aneurysm. Eur Rev Med Pharmacol Sci 2017;21(2):292-6.

6. Gaberel T, Borha A, di Palma C, Emery E. Clipping versus coiling in the management of posterior communicating artery aneurysms with third nerve palsy: A systematic review and meta-analysis. World Neurosurg 2016;87.

7. Wiebers DO, Whisnant JP, Huston J, et al. Unruptured intracranial aneurysms: natural history, clinical outcome, and risks of surgical and endovascular treatment. Lancet 2003;362(9378):103-110.

8. Yanovitch T, Buckley E. Diagnosis and management of third nerve palsy. Curr Opin Ophthalmol 2007;18(5):373-378

9. Mino M, Yoshida M, Morita T, Tominaga T. Outcomes of Oculomotor Nerve Palsy Caused by Internal Carotid Artery Aneurysm: Comparison between Microsurgical Clipping and Endovascular Coiling. Neurol Med Chir (Tokyo) 2015;55(12):885-890.

10. Zhang SH, Pei W, Cai XS, Cheng G. Endovascular management and recovery from oculomotor nerve palsy associated with aneurysms of the posterior communicating artery. World Neurosurg 2010;74(23):316-319.

11. Zu Q-Q, Liu X-L, Wang B, et al. Recovery of oculomotor nerve palsy after endovascular treatment of ruptured posterior communicating artery aneurysm. Neuroradiology 2017;59(11):1165-1170.

12. McCracken DJ, Lovasik BP, McCracken CE, et al. Resolution of Oculomotor Nerve Palsy Secondary to Posterior Communicating Artery Aneurysms: Comparison of Clipping and Coiling. Neurosurgery 2015;77(6).

13. Yerramneni VK, Chandra PS, Kasliwal MK, et al. Recovery of oculomotor nerve palsy following surgical clipping of posterior communicating artery aneurysms. Neurol India 2010;58(1):103-105.

14. Hall S, Sadek A-R, Dando A, et al. The Resolution of Oculomotor Nerve Palsy Caused by Unruptured Posterior Communicating Artery Aneurysms: A Cohort Study and Narrative Review. World Neurosurg 2017; 107:581-587.

15. Zheng F, Dong Y, Xia P, et al. Is clipping better than coiling in the treatment of patients with oculomotor nerve palsies induced by posterior communicating artery aneurysms? A systematic review and metaanalysis. Clin Neurol Neurosurg 2017;153:20-26.

16. Ahn JY, Han IB, Yoon PH, et al. Clipping vs coiling of posterior communicating artery aneurysms with third nerve palsy. Neurology 2006;66(1):121-123.

17. Wong GKC, Ng SC, Tsang PK, Poon WS.Clipping vs coiling of posterior communicating artery aneurysms with third nerve palsy. Neurology 2006;66(12).

18. Mansour N, Choudhari KA. Outcome of oculomotor nerve palsy from posterior communicating artery aneurysms: comparison of clipping and coiling. Neurosurgery 2007;60(3):E582; author reply E582.

19. Tan H, Huang G, Zhang T, Liu J, Li Z, Wang Z. A retrospective comparison of the influence of surgical clipping and endovascular embolization on recovery of oculomotor nerve palsy in patients with posterior communicating artery aneurysms. Neurosurgery 2015;76(6)

20. Yang M-q, Wang S, Zhao Y-1, Zhang D, Zhao J-z. Postoperative recovery from posterior communicating aneurysm complicated by oculomotor palsy. Chin Med J 2008;121(12):1065-1067.

21. Chang S-I, Tsai M-D, Wei C-P. Posterior communicating aneurysm with oculomotor nerve palsy: clinical outcome after aneurysm clipping. Turk Neurosurg 2014;24(2):170-173.

22. Hanse MCJ, Gerrits MCF, van Rooij WJ, Houben MPWA, Nijssen PCG, Sluzewski M. Recovery of posterior communicating artery aneurysminduced oculomotor palsy after coiling. AJNR Am J Neuroradiol 2008;29(5):988-990.

23. Engelhardt J, Berge J, Cuny E, Penchet G. Oculomotor nerve palsy induced by internal carotid artery aneurysm: prognostic factors for recovery. Acta Neurochir (Wien) 2015;157(7):1103-1111. 